\title{
BEHAVIOUR OF PARALLEL COUPLED MICROSTRIP BAND PASS FILTER AND SIMPLE MICROSTRIPLINE DUE TO THIN-FILM $\mathrm{AL}_{2} \mathrm{O}_{3}$ OVERLAY
}

\author{
S.B. RANE AND VIJAYA PURI \\ Department of Physics, Shivaji University, Kolhapur-416004 India \\ (Received July 28, 1995; in final form October 13, 1995)
}

\begin{abstract}
The X-band behaviour of a seven-section parallel-coupled microstrip band pass filter and microstripline due to thin-film $\mathrm{Al}_{2} \mathrm{O}_{3}$ overlay of different thickness is reported in this paper. This $\mathrm{Al}_{2} \mathrm{O}_{3}$ film can give a homogeneous overlay structure. There is a substantial increase in the bandwidth due to the overlay, the pass band extending towards higher frequency side. In most of the cases, an increase in the pass band transmittance of a microstripline also increases due to a thin-film $\mathrm{Al}_{2} \mathrm{O}_{3}$ overlay, especially for frequencies less than $9.0 \mathrm{GHz}$. At higher frequencies, random variations are observed. It is felt that thin-film overlays can be used to modify the microstripline circuit properties, thereby avoiding costly and time consuming elaborate design procedures.
\end{abstract}

\section{INTRODUCTION}

The need for less dispersion as well as greater coupling and resolution characteristics have lead to the study of bulk overlay ${ }^{1-4}$ microstrip structures. The stuck-on feature of bulk overlay is not desirable in applications involving environments like space, avionics, etc. Thick-film overlay ${ }^{5-7}$ provides a better alternative to bulk overlay but the thin-film overlay process is most compatible with the MIC's manufacturing process. The studies on use of thin film as an overlay on microstripline circuits are very limited ${ }^{8-11}$. Thin-film $\mathrm{Al}_{2} \mathrm{O}_{3}$ overlay structures can give a homogeneous overlay, i.e., overlay material and substrate material are the same.

This paper reports the effect of thin-film $\mathrm{Al}_{2} \mathrm{O}_{3}$ of different thickness on the characteristics of a seven-section parallel-coupled microstrip band pass filter and simple microstripline. There is a need for compact band pass filters with broad bandwidth characteristics. Since coupling takes place for nearly half the length of adjacent resonators, these types of filters can give large coupling for a given spacing between resonator strips. To the authors' knowledge, no studies on homogeneous thin-film overlay of thickness in the range of $0.1 \mu \mathrm{m}-2.0 \mu \mathrm{m}$ is available.

\section{EXPERIMENTAL}

A seven-section parallel-coupled microstrip band pass filter, as shown in figure 1, with a bandwidth of $600 \mathrm{MHz}(9.2-9.8 \mathrm{GHz})$ was designed according to Akhtarzad et $\mathrm{al}^{12}$ and Shamanna et al. ${ }^{13}$ The geometry of a single microstripline was according 


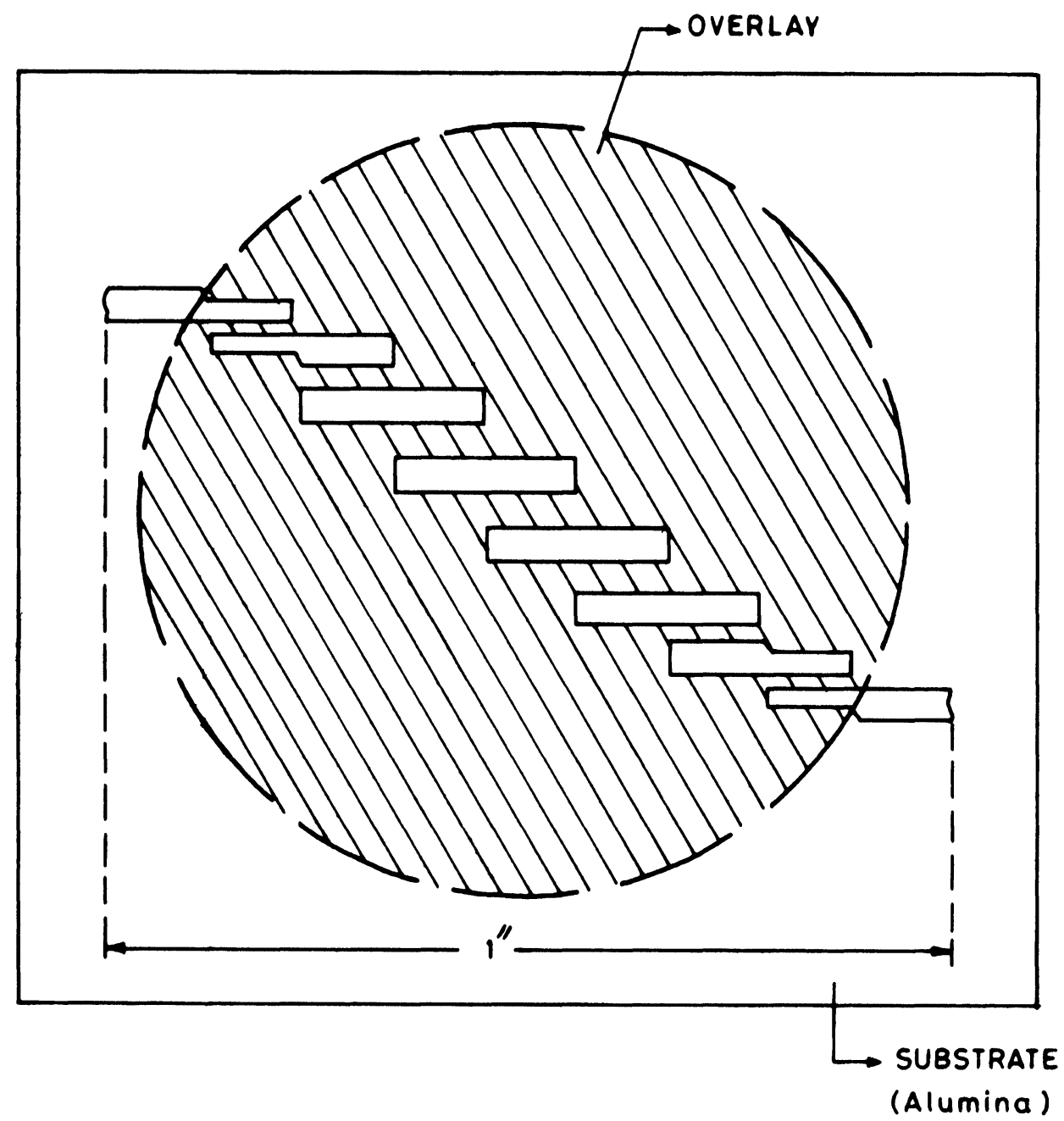

FIGURE 1 .Schematic of seven section parallel coupled microstrip band pass filter.

to standard design curves ${ }^{14}$. The width was $0.0625 \mathrm{~cm}$. The circuits were delineated photolithographically on $1 " \times 1 " \times 0.025^{\prime \prime}$ alumina substrates (Kyocera Japan) and metallized with copper by vacuum evaporation and electroplating. The thin-film overlay of $\mathrm{Al}_{2} \mathrm{O}_{3}$ of different thickness was deposited by a $3 \mathrm{KW}$ electron beam evaporation system under a vacuum of better than $10^{-5}$ Torr. The evaporation material was polycrystalline $\mathrm{Al}_{2} \mathrm{O}_{3}$ (Balzers $99.9 \%$ pure). The overlay was such that it completely covered the coupling regions of the filter (figure 1) and most of the microstripline. The microwave transmittance was measured point by point using a setup consisting of an X-band signal generator, directional coupler, and rf detector. As already reported ${ }^{7}$, the point to point measurements are within an error of $0.5 \mathrm{~dB}$ compared to network analyzer readings. The thickness of the overlay was gradually increased by repeated deposition, the microwave measurement being taken after each thickness increment. 


\section{RESULTS}

Figure 2 shows the plot of frequency vs transmittance of the seven-section band pass filter. Some representative overlay thicknesses are compared with, "without overlay" situation. The behavior of the bandwidth, transmittance at midband, and $3 \mathrm{db}$ down region due to thickness of the thin-film $\mathrm{Al}_{2} \mathrm{O}_{3}$ overlay is given in figure 3.

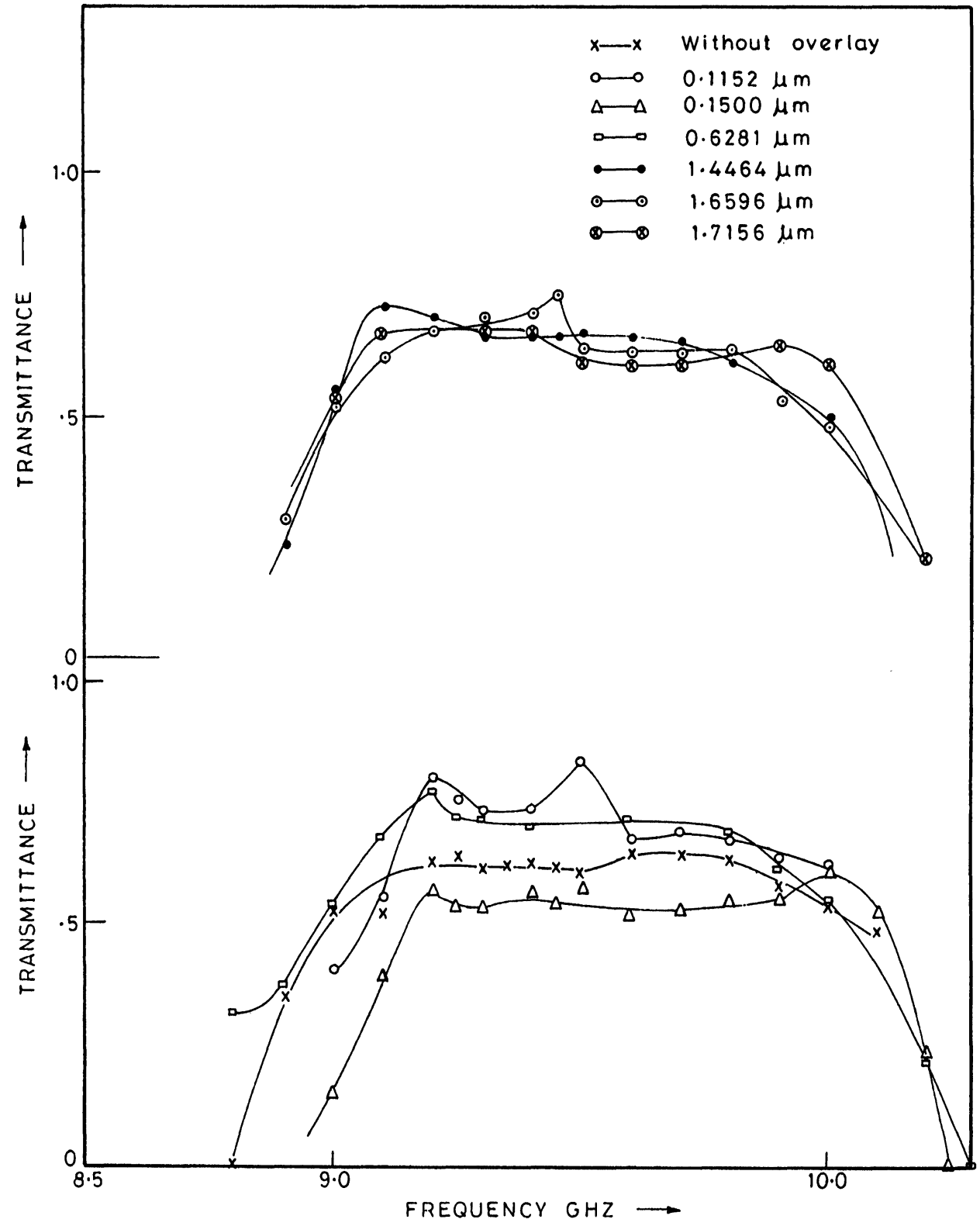

FIGURE 2 Transmission characteristics for the band pass filter without and with thin film $\mathrm{Al}_{2} \mathrm{O}_{3}$ overlay. 
From figure 2 , it is seen that, as expected, the filter without overlay has a bandwidth of $600 \mathrm{MHz}(9.2-9.8 \mathrm{GHz})$ with almost flat response in this region. Beyond this there is a gradual fall on both sides, though the transmittance goes to zero around $8.8 \mathrm{GHz}$ and $10.2 \mathrm{GHz}$. This might be due to fabrication tolerances and due to the fact that end effect correction were not made in the design. No post manufacture trimming was also undertaken. Our idea was to see the feasibility of effecting these corrections by means of a thin-film overlay.

With overlay of thin-film $\mathrm{Al}_{2} \mathrm{O}_{3}$, the shape of the filter response remains almost same with increasing bandwidth, i.e., in most of the cases, pass band extends to the higher frequency side. An increase in transmittance at pass band is also observed. Due to the overlay, some ripples are produced in the pass band region.

From figure 3, it is seen that for thicknesses of the overlay of $0.1152 \mu \mathrm{m}, 0.1700$ $\mu \mathrm{m}, 0.6281 \mu \mathrm{m}$, and above $1.4 \mu \mathrm{m}$, there is an increase in the midband transmittance; the maximum increase at $0.6281 \mu \mathrm{m}$. For a thickness of $0.1500 \mu \mathrm{m}$, a sharp decrease in transmittance is observed, though the bandwidth increases by $300 \mathrm{MHz}$. An overlay of $0.1500 \mu \mathrm{m}$ thickness seems to drastically effect the $3 \mathrm{db}$ transmission points also. Except for thicknesses of $0.700,1.2131$, and $1.5444 \mu \mathrm{m}$, the effect of all other thickness of overlay is to increase the bandwidth. All the curves drawn in figures 2 and 3 are an average of 6 samples.

The effect of the thickness from $1152 \AA-15456 \AA$ of the thin-film $\mathrm{Al}_{2} \mathrm{O}_{3}$ overlay on the transmittance of simple micro-stripline is depicted in figure 4 . The transmittance and reflectance data of the microstripline without overlay along with the reflectance due to some specific thickness of overlay are given in table 1. From the figure, it is seen that for frequency less than $9.0 \mathrm{GHz}$, there is an increase in transmittance due to the overlay except for thickness of $15244 \AA$. Beyond $9.0 \mathrm{GHz}$ there is a thickness dependent behavior without any specific trend, although at around $9.5 \mathrm{GHz}$ a peaking tendency is observed. At a thickness of $3041 \AA$ and $14896 \AA$, an increased transmission coefficient is obtained at higher frequencies. No definite relation could be obtained between thickness of overlay and transmittance, although in general it can be stated that the $\mathrm{Al}_{2} \mathrm{O}_{3}$ thin-film overlay has the effect of increasing the transmission properties of the simple microstripline although an oscillatory behavior is observed. Joshi et al ${ }^{11}$ observed increased transmittance due to a CVD deposited $\mathrm{Al}_{2} \mathrm{O}_{3}$ thin film of thickness around $3000 \AA$. These authors deposited only one thickness. From Table 1, it is observed that there is not much change in reflectance due to overlay especially below $9.0 \mathrm{GHz}$, and random behavior is obtained above $9.0 \mathrm{GHz}$, although higher thickness seems to lower the reflectance at higher frequency.

TABLE 1. Data of reflectance of microstripline without and with thin film $\mathrm{Al}_{2} \mathrm{O}_{3}$ overlay.

\begin{tabular}{llllllll}
\hline $\begin{array}{l}\text { Frequency } \\
\text { GHz }\end{array}$ & $\begin{array}{c}\mathrm{T}^{2} \\
\text { without } \\
\text { overlay }\end{array}$ & $\begin{array}{c}\text { Without } \\
\text { overlay } \\
\rho^{2}\end{array}$ & 1152 & 1500 & 3041 & 7731 & 14896 \\
\hline 8.0 & 0.77 & 0.04 & 0.04 & 0.03 & 0.05 & 0.04 & 0.04 \\
8.5 & 0.79 & 0.13 & 0.13 & 0.18 & 0.15 & 0.12 & 0.10 \\
9.0 & 0.74 & 0.11 & 0.02 & 0.02 & 0.05 & 0.11 & 0.21 \\
9.5 & 0.69 & 0.23 & 0.11 & 0.14 & 0.17 & 0.18 & 0.26 \\
10.0 & 0.71 & 0.12 & 0.20 & 0.24 & 0.21 & 0.16 & 0.04 \\
10.5 & 0.67 & 0.05 & 0.15 & 0.10 & 0.02 & 0.02 & 0.001 \\
\hline
\end{tabular}




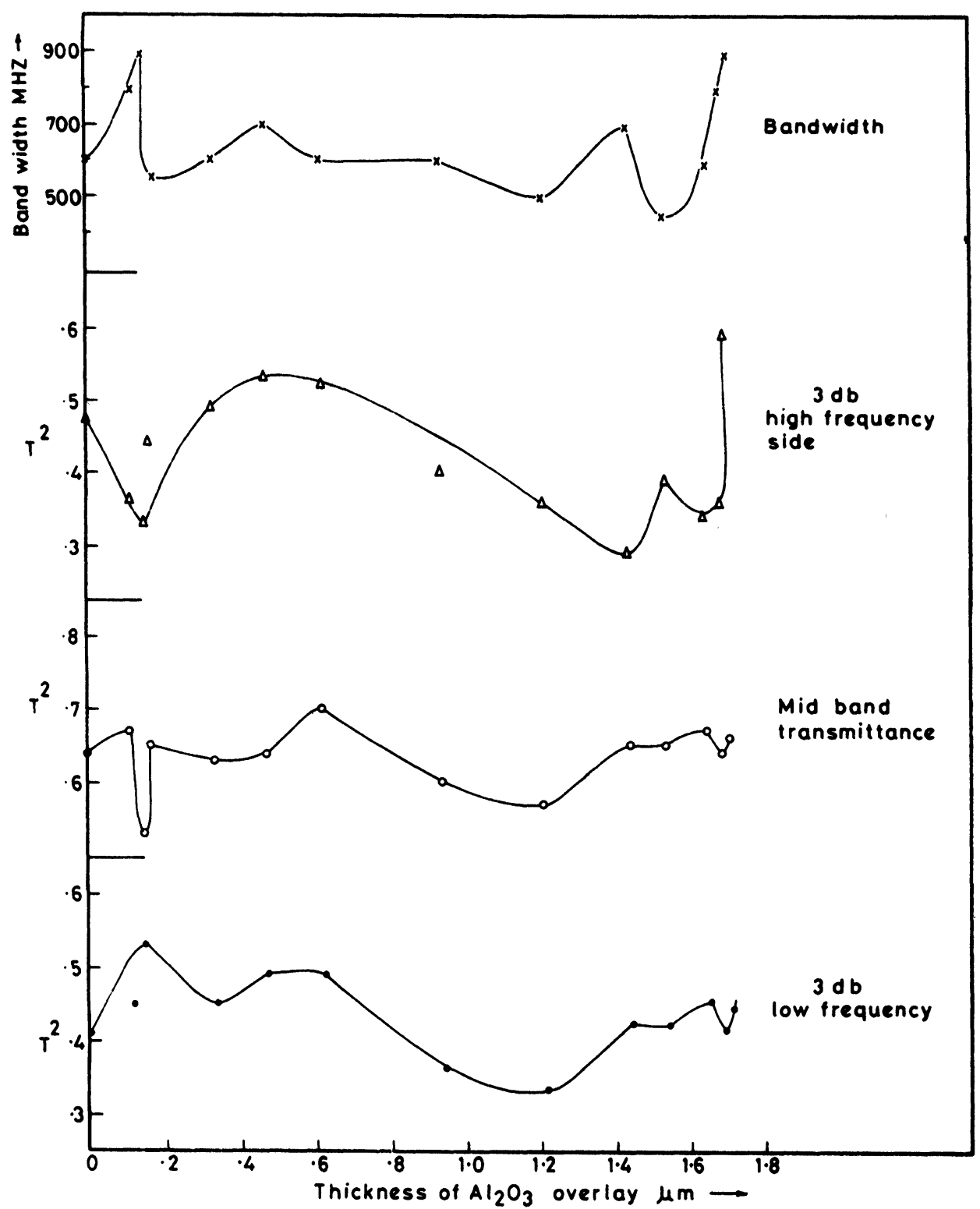

FIGURE 3 Transmittance versus thickness of overlay at midband, $3 \mathrm{~dB}$ regions and for band width.

From the above results, it can be emphatically stated that the thin-film $\mathrm{Al}_{2} \mathrm{O}_{3}$ overlay does cause changes in the behavior of both the band pass filter and simple microstripline.

\section{DISCUSSION}

The $\mathrm{Al}_{2} \mathrm{O}_{3}$ thin film being a homogeneous overlay (material same as substrate), the conditions of permittivity are almost the same above and below the microstripline 


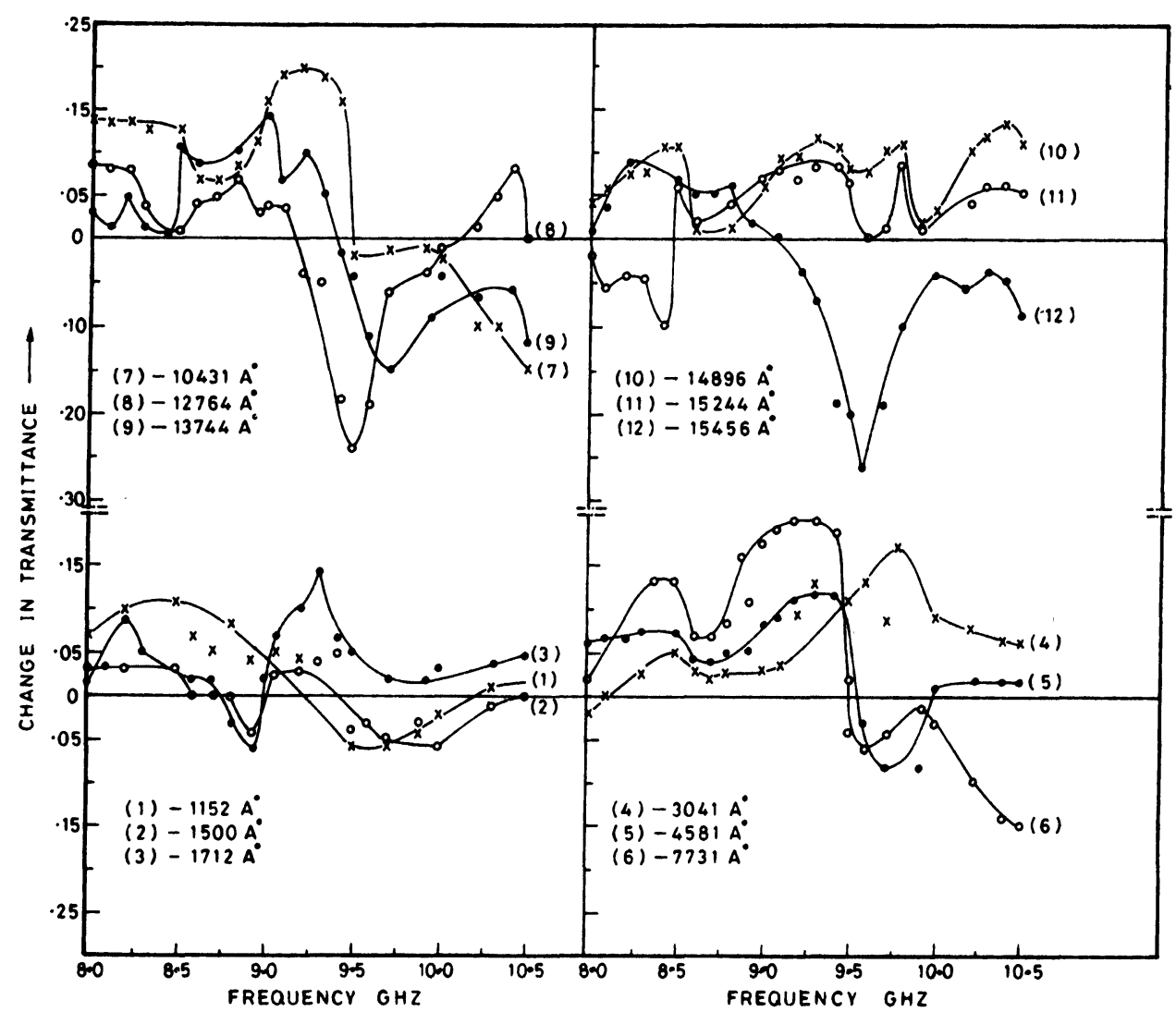

FIGURE 4 Change in transmittance of microstripline as a function of frequency for various $\mathrm{Al}_{2} \mathrm{O}_{3}$ thin film overlay thickness.

circuits. The fields associated with the dominant mode would interact with the overlay thereby changing the transmission characteristics of the circuits. The higher-order modes with their fields spread over the circuit might also be suppressed resulting in an increased transmission coefficient probably due to reduction in radiation loss. If all the spurious modes were suppressed, then one would expect a dispersionless output for the microstripline. Our results show (figure 4) that for some thickness of overlay the dispersion has increased. For higher thickness of overlay $>1.4 \mu \mathrm{m}$ especially for higher frequency, the reflection coefficient also decreases (Table 1) along with an increase in transmission coefficient indicating an improvement in impedance matching. The extent of interaction of the overlay material with the fields above the microstripline seems to be highly dependent on the frequency and thickness of the thin-film overlay while some additional dielectric related surface modes are introduced.

The bandwidth of the parallel-coupled filter is sensitive to changes in the coupling especially of the first and last coupling structure. Since the overlay is in the thin film form, it will be deposited uniformly in between the coupling structures as well as over the conductor structures of the filter. The overlay might be changing the impedance of the coupling structures. The thickness of the conductor is $\sim 4 \mu \mathrm{m}$, whereas maximum overlay thickness is $\sim 1.7 \mu \mathrm{m}$. That means between the two 
parallel-coupled resonating structures, there is a discontinuity in the dielectric medium causing changes in the impedances of the circuit. This might cause change in the pass band of the filter. Also although the physical lengths remain the same, the guide wavelength might be changing because of the addition of dielectric material in between the coupling region. Our XRD and dielectric constant of data of both substrate and thin-film $\mathrm{Al}_{2} \mathrm{O}_{3}$ shows a weak crystalline order for electron beam deposited $\mathrm{Al}_{2} \mathrm{O}_{3}$ with a dielectric constant of $\sim 7.2$, whereas the alumina substrate is highly polycrystalline having a dielectric constant of $\sim 10.0$.

It has been reported ${ }^{15}$ that an increase in the dimensions of the first and last coupled section reduces the bandwidth. These type of parallel coupled filters have radiative loss due to the ends of the resonating structure being open. The overlay material might also be suppressing these unwanted fields from the edges. Since the variation in transmittance is highly overlay thickness dependent, it is felt that some complex field distribution comes into effect due to overlay, which is mainly an oxide layer effect. These might also be causing variations of lumped capacitance, edge capacitance, and open circuit end effects. Since seven coupling sections and 14 ends are involved, the interaction becomes more complex.

It is felt that a combination of various factors contribute to the behavior of the seven-section microstrip band pass filter and microstrip line. It would be interesting to study the effect of overlay in thin film form when thickness becomes equal to conductor thickness. Further work in this direction is in progress.

\section{CONCLUSION}

It is felt that thin-film overlays even in the $1000 \AA$ range can be used to modify or improve the properties of microstripline circuits. Use of a homogeneous overlay simplifies the design procedures, as the dielectrics above and below the circuit are the same. The variations in the properties of the band pass filter by changing the number of coupling structures and overlaying with a wide range of thickness of $\mathrm{Al}_{2} \mathrm{O}_{3}$ from thin film to pellet needs to be studied in detail both experimentally and theoretically. It is felt that some particular thickness and particular materials, when used as overlay over the circuits, will enable one to achieve highly accurate properties without costly, time consuming design procedures.

\section{ACKNOWLEDGEMENT}

One of the authors Vijaya Puri gratefully acknowledges the award of research scientist by UGC India.

\section{REFERENCES}

1. B. Sheleg, B.E. Spielmann; IEEE Trans MTT 22, 1216 (Dec 1974).

2. K. Chang, J. Klein, Electron Lett. 23, 535 (1987).

3. S.K. Koul, Int. J. Electronics 70, 447 (1991).

4. F.C. Silva, S.B.A. Fonseca, A.J. Martino, A.J. Giarola, IEEE MW and Guided wave Lett 2, 359 (Sep 1992).

5. S.M.V. Iyer, R.N. Karekar, Electron Lett 28, 873 (23 April 1992). 
6. A.M. Joshi, M.M. Mandhre, M.L. Jadhav, S.A. Gangal, R.N. Karekar, Jpn. J Appl Phys 27, 2168 (Nov 1988).

7. V. Puri, Hybrid Circuits 35, 12 (Sep 1994).

8. R.N. Karekar, M.K. Pande, IEEE Trans MTT 24, 262, (1976).

9. M.K. Pande, R.N. Karekar, IEEE Trans MTT 25, 74 (1977).

10. K. Vijaya, M.L. Jadhav, S.A. Gangal, R.N. Karekar, Bull Mater Sci 8, 397 (June 1986).

11. K.K. Joshi, C. Dhanavantri, S.A. Gangal, R.N. Karekar, Bull Mater Sci 8, 535 (Oct 1986).

12. S.Akhtarzad, T.R. Rowbatham, P.B. Johns, IEEE Trans MTT 23, 286 (1975).

13. K.N. Shamanna, V.S. Rao, S.P. Kosta, Int. J. Electronics 45, 89 (1978).

14. T.G. Bryant, A.J. Weiss, IEEE Trans MTT 16, 1021 (1968).

15. K.K. Pang, IEEE Trans MTT 21, 607 (Oct 1973). 

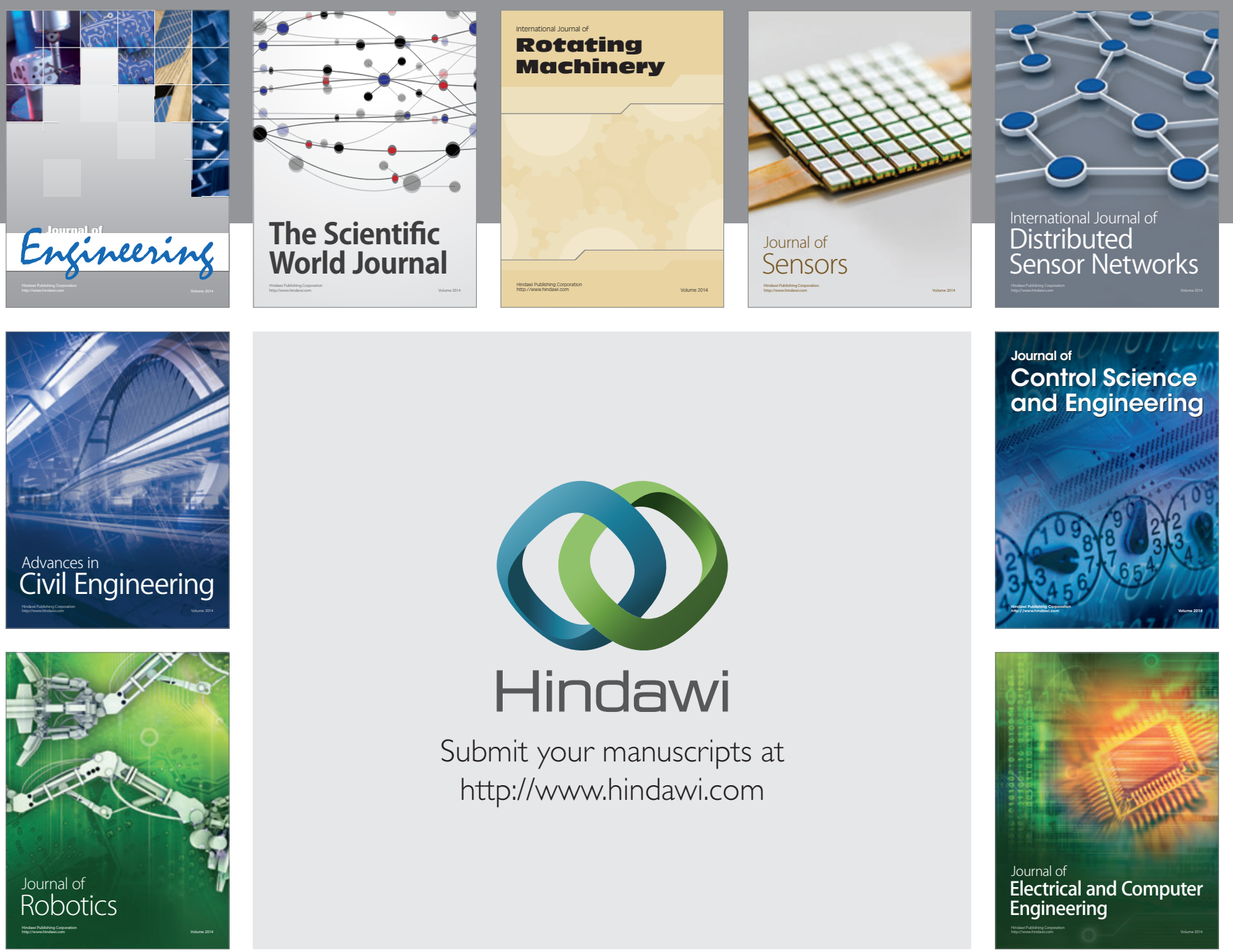

Submit your manuscripts at

http://www.hindawi.com
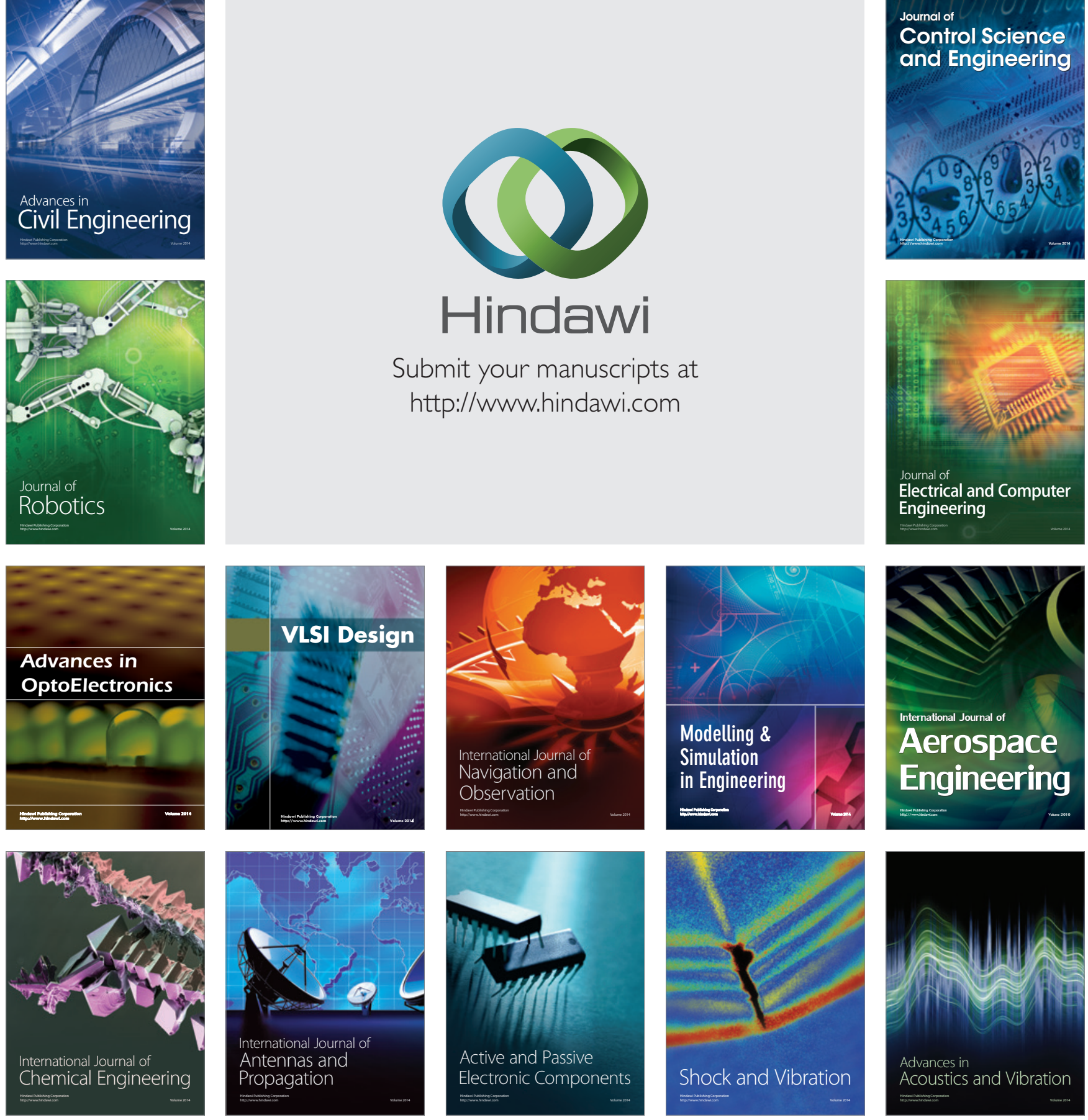